\title{
A method for determination in situ of variations within the hepatic lobule of hepatocyte function and metabolite concentrations
}

\author{
Shamus P. BURNS*, Robert D. COHEN*\|, Richard A. ILES*, Jocelyn P. GERMAIN $\dagger$, Thomas C. H. GOING*, Stephen J. W. EVANS $\$$ \\ and Patrick ROYSTON $\S$ \\ ${ }^{*}$ Medical Unit, †Department of Oral Pathology and †Department of Epidemiology and Medical Statistics, St. Bartholomew's \\ and The Royal London Hospital School of Medicine and Dentistry (Queen Mary and Westfield College, University of London), Turner Street, London E1, \\ and §Department of Medical Statistics, The Royal Postgraduate Medical School, Hammersmith Hospital, London W12 0NN, U.K.
}

\begin{abstract}
A method is described for the production of detailed maps of intralobular variations of hepatocyte function and metabolite concentrations, based on variable destruction by digitonin of the lobule from the centrilobular direction. Instead of the conventional approach, in which isolated hepatocytes are then prepared and studied in suspension, perfusion is continued after digitonin treatment and the function of the unaffected lobular remnants is determined, or mean metabolite concentrations are measured by ${ }^{31} \mathrm{P}-\mathrm{NMR}$. These measurements are plotted against the degree of destruction, determined precisely after each study by automated quantitative histomorphometry. These plots are transformed into curves of the function or metabolite concentration of nominal single cells at any point along the radius of the lobule. Gluconeogenesis from lactate remained stable,
\end{abstract}

although reduced, even after $85-90 \%$ lobular destruction, predominated periportally and disappeared by $50 \%$ along the radius of the lobule. In ${ }^{31} \mathrm{P}-\mathrm{NMR}$ studies, employing $1.5 \mathrm{mM}$ lactate as substrate, narrowing of the intracellular $\mathrm{P}_{i}$ resonance was observed as digitonin destruction increased; this was attributed to a decrease in the intralobular heterogeneity of the intracellular $\mathrm{pH}$, which fell from approx. 7.9 to $<7.4$ along the first $16 \%$ of the lobular radius (from the periportal end) and to $<7.3$ in the remainder of the lobule. The ATP concentration rose, and then fell, along the radius of the lobule in a centripetal direction. The method is potentially generally applicable to a wide range of hepatocellular functions and to the measurement of metabolite concentrations, most conveniently those susceptible to estimation by NMR.

\section{INTRODUCTION}

Heterogeneity of hepatocyte function along the length of the sinusoid is well established [1]. Quistorff and Grunnet [2] showed that short periods of perfusion of rat liver with digitonin could selectively destroy the periportal zone of the lobule if perfused in the antegrade direction, or the centrilobular zone if perfused retrogradely. In the short time in which it was in contact with the liver cell, the digitonin was immediately and almost quantitatively titrated by the plasma membrane, so that if the digitonin was rapidly flushed out by digitonin-free medium in the reverse direction, cells distal to the point reached by the digitonin front were unaffected. There is an extremely sharp demarcation (one cell to the next) between the cells affected by digitonin and those not affected [3,4]. Several groups [5-8] then prepared isolated hepatocytes in the usual way from the remnant after digitonin perfusion, and were able to demonstrate variations in metabolic function according to the zone of origin of the isolated cells. Gluconeogenesis, glycogen synthesis and ureogenesis were mainly found in cells from the periportal zone, whereas glutamine synthesis was located in cells from the centrilobular zone. No zonation of glycolysis is apparent using the digitonin technique [8], although a predominantly centrilobular localization has been suggested from microdissection techniques [9].

However, it has not been possible using this digitonin technique to plot continuous curves of the distribution of metabolic functions, intracellular $\mathrm{pH}\left(\mathrm{pH}_{\mathrm{i}}\right)$ and concentrations of metabolites such as adenine nucleotides along the radius of the hepatic lobule. In the present study, we describe a method for achieving these aims in rat isolated perfused liver, without the need to prepare isolated hepatocytes and thus eliminating the attendant uncertainties about the effects of cell isolation, including epithelial disruption and destruction of vascular relationships. We give examples of the use of the method to determine the distribution under defined conditions of gluconeogenesis from lactate, $\mathrm{pH}_{\mathrm{i}}$, inorganic phosphate $\left(\mathrm{P}_{\mathrm{i}}\right)$ and ATP. The method is, however, potentially generally applicable to a wide range of hepatic functions and metabolite concentrations, most easily those that can be determined by NMR spectroscopy.

The method is based on observations on the post-digitonin intact remnant of the liver, perfused in the antegrade direction, rather than on studies of isolated hepatocytes prepared after digitonin perfusion. By perfusing in the retrograde direction with digitonin for a period which varies between livers, the function of different-sized unaffected lobular remnants with respect to the process under study can be plotted against the remnant volume. The latter is measured by preparing histological sections of the liver at the end of the observations; accurate measurements of the proportion remaining unaffected are then obtained using automated quantitative histomorphometry.

\section{EXPERIMENTAL}

\section{Materials}

L(+)-Lactic acid was obtained from Sigma Chemical Co. (Poole, Dorset, U.K.), methylenediphosphonate (MDP) was from Goss Scientific Instruments, and digitonin was from BDH. All other

Abbreviations used: MDP, methylenediphosphonic acid; FVR, fractional volume remaining; $\mathrm{pH}_{\mathrm{i}}$, intracellular $\mathrm{pH}$.

\| To whom correspondence should be addressed: Medical Unit, The Royal London Hospital, Whitechapel Road, London E1 1BB, U.K. 
chemicals were of the highest analytical grade available commercially.

\section{Experimental animals}

Male Wistar rats (260-340 g), fed on standard rat cake and then starved for $48 \mathrm{~h}$, were used for all studies. Isolated liver perfusions were established at $36.5^{\circ} \mathrm{C}$ by a modification of a previously described method [10], but omitting erythrocytes and albumin and increasing the flow rate to $11 \mathrm{ml} / \mathrm{min}$ per $100 \mathrm{~g}$ body weight. The perfusate was Krebs-Henseleit bicarbonate buffer [11] gassed with $95 \% \mathrm{O}_{2} / 5 \% \mathrm{CO}_{2}$ to give $\mathrm{pH} 7.4$; portal and hepatic venous $\mathrm{PO}_{2}$ values were typically 70 and $20 \mathrm{kPa}$ respectively. In experiments involving NMR, this degree of oxygenation was ensured by a non-metallic oxygenator close to the spectrometer, the pump and reservoir being sited outside the $5 \mathrm{G}(0.5 \mathrm{mT})$ line.

Two types of studies were carried out. The first (bench studies) were aimed at determining the relationship between the degree of destruction and rate of gluconeogenesis from saturating concentrations $(6 \mathrm{mM})$ of lactate. For the second type (NMR studies), changes in $\mathrm{pH}_{\mathrm{i}}, \mathrm{P}_{\mathrm{i}}$ and ATP with respect to the degree of destruction were studied using $1.5 \mathrm{mM}$ lactate. The latter concentration is below the saturating concentration for gluconeogenesis from lactate.

\section{Bench studies}

$\mathrm{L}(+)$-Lactate $(6 \mathrm{mM})$ was used as substrate. After a $20 \mathrm{~min}$ stabilization period, two pairs of baseline portal and hepatic venous samples were taken with simultaneous flow rate measurements. Digitonin (4 mg/ml in Tris buffer, $\mathrm{pH} \mathrm{7.4)}$ was then perfused retrogradely at $30 \mathrm{ml} / \mathrm{min}$ for various times (not exceeding $2 \mathrm{~min}$ ) in different livers. In this way a range of degrees of destruction varying from 15 to $95 \%$ in different livers, depending roughly on the digitonin perfusion times, was achieved. Perfusion was then re-established in the antegrade direction. The perfusion pressure was somewhat raised for 1-2 min immediately post-digitonin, but then returned to its previous value. The liver was allowed to stabilize for 10-15 min, and a further series of three portal-hepatic venous sample pairs were then taken at 10 min intervals. Control observations were made in preparations in which digitonin-free buffer was perfused retrogradely for $2 \mathrm{~min}$. Glucose was measured in the samples [12] and glucose production calculated by the Fick principle. After these observations, the liver was fixed by perfusion with $30 \mathrm{ml}$ of $10 \%$ formalin over $1 \mathrm{~min}$. Blocks were then removed for histology and stored in formalin.

\section{NMR studies}

${ }^{31} \mathrm{P}-\mathrm{NMR}$ spectra were obtained with a Sisco $4.7 \mathrm{~T}$ spectrometer; the magnet had a horizontal bore of diameter $220 \mathrm{~mm}$. A twoturn surface coil of radius $11 \mathrm{~mm}$ was placed directly on the liver surface and tuned to $81 \mathrm{MHz}$. Spectra were collected using hard $90^{\circ}$ pulses, arranged to maximize the liver signal, of $70 \mu \mathrm{s}$ duration with a $6 \mathrm{kHz}$ sweepwidth, and an acquisition time of $0.3 \mathrm{~s}$ with no relaxation delay, producing a recycling time of $0.3 \mathrm{~s}$. This rate of pulsing maintains saturation of the $P_{i}$ signal from the extracellular space. Spectra were processed using $5 \mathrm{~Hz}$ exponential line broadening, and referenced to both the $\alpha$-ATP $+\alpha$-ADP (-7.57 p.p.m.) and the MDP (approx. 20.95 p.p.m.) resonances. The MDP resonance was generated from a capsule containing $110 \mathrm{mM}$ MDP placed below the liver. The MDP chemical shift varied by less than 0.04 p.p.m. during individual perfusions, but could vary significantly between perfusions; the initial referencing of the MDP resonance to the resonance at -7.57 p.p.m. was therefore necessary. The MDP peak was particularly important at high degrees of destruction, when the ATP peak was low. Chemical shifts of the $P_{i}$ peak were measured by a computer program which removes subjectivity, and the $\mathrm{pH}_{\mathrm{i}}$ thus derived. Peaks were quantified by area, estimated by cutting and weighing. After initial shimming and collection of baseline spectra, the liver was removed from the spectrometer and perfused with digitonin or control buffer in the retrograde direction as for the bench experiments. The preparation was then replaced in the spectrometer and re-shimmed within $15 \mathrm{~min}$. The accumulation time for a single spectrum was $10 \mathrm{~min}$, and observations were made immediately before digitonin treatment and $30-40 \mathrm{~min}$ later. The liver was then fixed as above and blocks taken for histology.

\section{Quantitative histomorphometry}

The blocks were embedded in paraffin wax, and $5 \mu \mathrm{m}$ sections were stained with haematoxylin and eosin. Measurements of the ratio of the area affected by digitonin to the total area of the liver section were made using Seescan quantitative histomorphometry equipment. At $10 \times$ magnification, five fields, each containing approximately five lobules, were scanned per section. Allowances could be made for the small area occupied by sinusoidal spaces and vasculature.

\section{Derivation of plots}

The fractional volume of viable liver remaining unaffected (FVR), and thus the mean fractional lobular volume remaining, was derived by raising the measured fractional area destroyed by the power of $3 / 2$ and subtracting from unity. The fractional function of the liver remaining unaffected (e.g. gluconeogenesis from lactate) was obtained by dividing the mean of the post-digitonin observations by the mean of the pre-digitonin observations and then plotted against the FVR. The mean fractional concentrations of ${ }^{31} \mathrm{P}-\mathrm{NMR}$-detectable metabolites $\left(\mathrm{P}_{\mathrm{i}}, \mathrm{ATP}\right)$ in the liver remaining unaffected were calculated in the same way and plotted against the FVR. The mean $\mathrm{pH}_{\mathrm{i}}$ in the remaining unaffected liver was also plotted against the FVR. For $\mathrm{P}_{i}$, a straight line was fitted to the data. For gluconeogenesis and ATP, simple non-linear curves $f(x)$ were fitted to the data, where $x$ denotes the FVR. For ATP, $\mathrm{P}_{\mathrm{i}}$ and gluconeogenesis, curves were chosen to pass through the $(1,1)$ point, i.e. that at which zero destruction by digitonin has been effected and therefore no change in function or metabolite concentration has occurred; justification for this assumption is provided in the Results section. The curve chosen for ATP was a two-parameter exponential function of the form $f(x)=\exp \left[b\left(1-c^{x-1}\right)\right]$; for gluconeogenesis the curves were of the form $f(x)=1+b\left(1-c^{x-1}\right)$.

The data and fitted curves of the above-described plots refer to the function (or average metabolite concentration) of the whole of the remaining viable liver, i.e. periportal to the demarcation between viable liver and digitonin-affected cells. In order to plot the function (or metabolite concentration) at each point along the lobular radius, the mathematical transformation of the fitted curves described in Appendix 1 was employed, assuming a spherical model for the lobule. Plots obtained in this way are shown in Figure 3. This representation would, for example, facilitate comparison with histochemical or histological data. Appendix 2 demonstrates how the $95 \%$ confidence limits of the derivative curves of Figure 3 were obtained.

Results are given as means \pm S.E.M. 


\section{RESULTS}

\section{Histomorphometry}

The extremely sharp demarcation between digitonin-affected and unaffected tissue as seen in photographs published by Quistorff and Romert [3] was confirmed. Instead of staining with Toluidine Blue, we used haematoxylin and eosin; this gave slightly greater



Figure 1 Relative rates of gluconeogenesis (compared with pre-destruction mean) from lactate $(6 \mathrm{mM})$ at different degrees of retrograde destruction

Each curve shows the means \pm S.E.M. of observations in three rats. At the arrow, digitonin (4 mg/ml in Tris buffer, $\mathrm{pH} 7.4$ ) was perfused retrogradely for various periods. The FVRs in the three groups were as follows: $\diamond, 0.82-0.89 ; 0,0.26-0.37 ; \square, 0.17-0.24$.

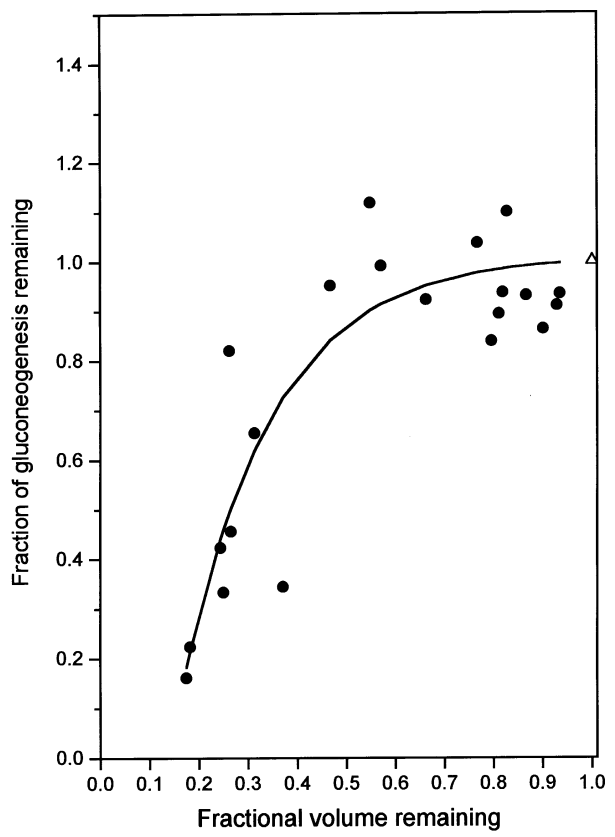

reproducibility of repeated estimations of the fractional area remaining, which was $2.8 \%(n=10)$.

\section{Control studies}

In these studies, in which no digitonin was added to the retrograde perfusion fluid (see the Experimental section), the post-/preretrograde perfusion ratios were as follows: gluconeogenesis (6 mM lactate), $1.03 \pm 0.02, n=9$; gluconeogenesis $(1.5 \mathrm{mM}$ lactate), $1.01 \pm 0.05, \quad n=7 ; \quad \mathrm{P}_{\mathrm{i}}, \quad 1.01 \pm 0.04, \quad n=7 ; \quad$ ATP, $0.99 \pm 0.04, n=7 ; \mathrm{pH}_{\mathrm{i}},-0.01 \pm 0.02, n=7$. None of these ratios or differences was significantly different from unity, justifying the assumption of the $(1,1)$ point as detailed in the Experimental section.

\section{Bench studies}

Figure 1 shows rates of glucose production from $6 \mathrm{mM} \mathrm{L}(+)$ lactate at three levels of liver destruction. Digitonin was perfused in the retrograde direction for periods ranging from 20 to $120 \mathrm{~s}$. It may be seen that glucose production rates were relatively stable between 10 and $30 \mathrm{~min}$ post-digitonin. The absolute glucose production rate pre-digitonin was $1.73 \pm 0.11 \mu \mathrm{mol} / \mathrm{min}$ per $100 \mathrm{~g}$ body weight $(n=21)$. Analysis of successive glucose production rates post-digitonin for all studies by the method of summary statistics [13], using the linear regression coefficient as the summary statistic, showed that the new rate of gluconeogenesis $10 \mathrm{~min}$ after digitonin treatment (dependent on level of destruction) was increased on average by $4 \%$ at $30 \mathrm{~min}$ following digitonin treatment. However, this increase was not significant, indicating that the hepatocytes in the remnant liver are stable. More limited data suggest that this stability persists for at least $60 \mathrm{~min}$.

Figure 2 (left panel) is a plot of glucose production in 21 individual livers as a percentage of control against the FVR. No



Figure 2 Fraction of gluconeogenesis remaining from $6 \mathrm{mM}$ (left panel) and $1.5 \mathrm{mM}$ (right panel) lactate, plotted against average FVR after retrograde digitonin perfusion

Gluconeogenic rates are given relative to the pre-digitonin rate. Each point is derived from a single liver. Left panel: the solid line represents $y=1+0.009096\left(1-0.004249^{\mathrm{FVR}-1}\right)$. Right panel: the curve represents $y=1+0.03893\left(1-0.03685^{\mathrm{FVR}-1}\right)$. Both fitted curves pass through the point $(1,1)$, denoted by $\triangle$. 



Figure 3 Effect of fractional radius from lobular centre on gluconeogenesis and metabolite concentrations

Upper panel: relative gluconeogenic function (Gng) from $1.5 \mathrm{mM}$ lactate, and $\mathrm{P}_{\mathrm{i}}$ and ATP concentrations, plotted against fractional radius $r$ from the lobular centre, derived (see Appendix 1) from eqn. 3 (gluconeogenesis) and eqn. 4 (metabolite concentrations) using the fitted curves in Figures 2, 5 and 6 . The same studies as in Figure 2 (right panel) are shown. Values are plotted as a fraction of the extreme or near-extreme periportal value (see Appendix 1). The $95 \%$ confidence intervals (see Appendix 2) for the curves are indicated by the fainter lines. Note that: (i) the abscissa declines in value from left to right, (ii) $r=0.4$ represents a fractional volume destroyed of only 0.064 (FVR $=0.936$ ), and (iii) the confidence limits narrow in the region corresponding to $r \sim 0.8$ (fractional volume $\sim 0.5$ ). Lower panel: relative gluconeogenesis from $6 \mathrm{mM}$ lactate (derived from data in Figure 2, left panel) compared with that from $1.5 \mathrm{mM}$ lactate (taken from the upper panel).

clear fall in glucose production occurred until more than $70 \%$ of the lobular volume had been destroyed. The fitted curve has been used for the plot in Figure 3 (lower panel), using eqn. (3) in

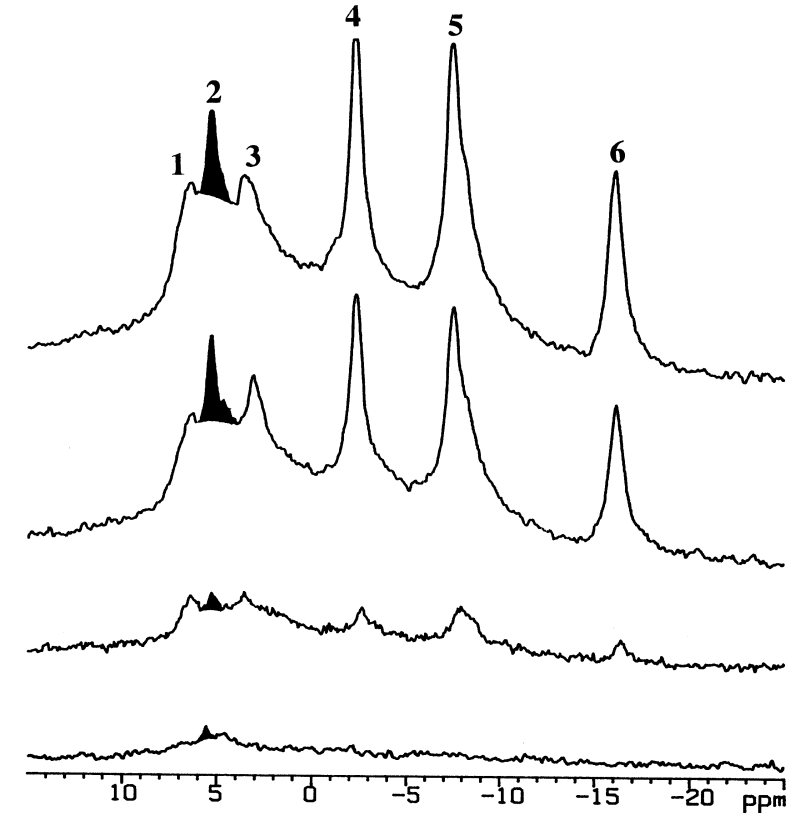

Figure $4 \quad{ }^{31} \mathrm{P}-\mathrm{NMR}$ spectra from livers perfused with lactate $(1.5 \mathrm{mM})$ and subject to retrograde destruction

Fractional volume destructions were $0,0.33,0.78$ and 0.96 (from top to bottom). Observations were made over the period 15-25 min after digitonin or control retrograde perfusion. Peak assignments: 1 , phosphomonoesters; $2, \mathrm{P}_{\mathrm{i}}$; 3 , phosphodiesters; $4-6, \gamma-, \alpha$ - and $\beta$-ATP respectively. The shaded areas were used for estimation of $P_{i}$ concentration.

Appendix 1 to derive the relationship between radial distance from the lobular centre and the gluconeogenic function of a nominal single cell located at that radial distance, compared with the function of a nominal single cell at the extreme periportal end of the lobular radius. Thus Figure 3 (lower panel) demonstrates that, under these conditions, gluconeogenesis from lactate predominates in the periportal zone and disappears by about $50 \%$ of the distance along the lobular radius.

\section{${ }^{31}$ P-NMR studies}

Figure 4 shows spectra from a series of preparations that have undergone various degrees of destruction by digitonin. In these studies, $1.5 \mathrm{mM}$ lactate was the substrate. The absolute predigitonin rate of glucose formation was $1.45 \pm 0.14 \mu \mathrm{mol} / \mathrm{min}$ per $100 \mathrm{~g}(n=16)$, with again no significant drift in the postdigitonin values as assessed by summary statistics [13]. The plot of gluconeogenesis remaining against FVR is shown in Figure 2 (right panel), and the transformed plot of function against position in lobule is shown in Figure 3. Comparison of the primary data for the two substrate concentrations $(6 \mathrm{mM}$ and $1.5 \mathrm{mM}$ lactate; Figure 2, left and right panels respectively) by a form of analysis of variance, using a quadratic fit, indicates that neither the quadratic nor linear terms were significantly different. The difference in the constant term just failed to reached significance $(P=0.056)$. The consequence is that the derived curves (Figure 3, lower panel) are not significantly different.

\section{$P_{\mathrm{i}}$}

It was possible to quantify $\mathrm{P}_{\mathrm{i}}$ (intracellular) after liver destruction as great as $90-95 \%$ because, with increasing degree of destruction, the width of the $\mathrm{P}_{\mathrm{i}}$ resonance diminished, thereby 


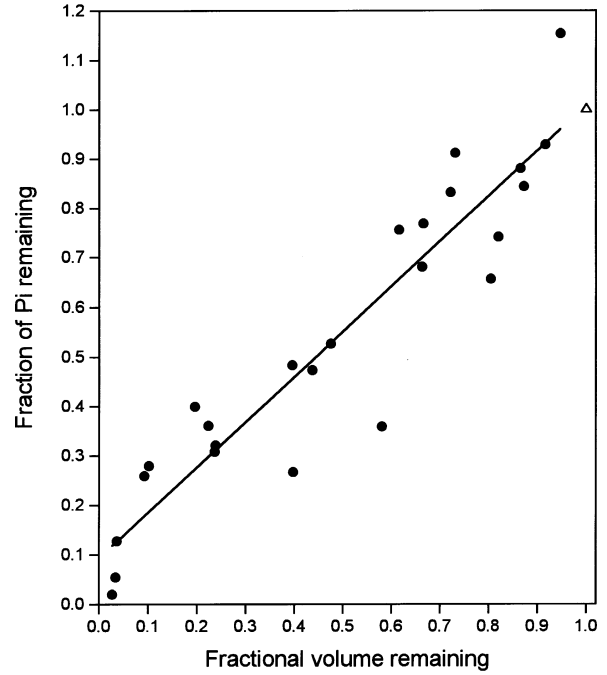

Figure 5 Intracellular $P_{i}$ remaining, plotted against FVR

The substrate was $1.5 \mathrm{mM}$ lactate. Symbols are as in Figure 2. The solid line represents $y=0.0938+0.914$ FVR. Data are from the same studies as in Figure 2 (right panel).

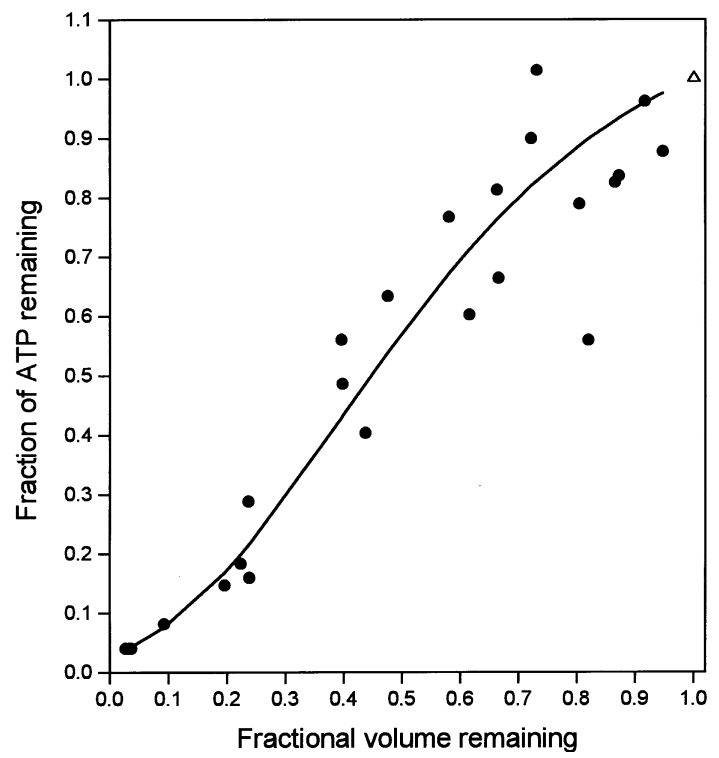

Figure 6 Fraction of ATP remaining, plotted against FVR

The substrate was $1.5 \mathrm{mM}$ lactate. Symbols are as in Figure 2. The two points at the smallest FVR have been plotted midway between zero and 0.09 fractional ATP remaining, since below the latter level ATP was not measurable. The curve represents $y=$ $\exp \left[0.1279\left(1-0.03469^{\mathrm{FVR}-1}\right)\right]$. The denominator in eqn. (4) of Appendix 1 was calculated at $\mathrm{FVR}=0.05$. Data are from the same studies as in Figure 2 (right panel).

improving resolution. We attribute the narrowing to a progressive decrease in $\mathrm{pH}_{\mathrm{i}}$ heterogeneity as destruction increases (see below). Figure 5 is a plot of fractional $P_{i}$ remaining against the FVR. There was a progressive diminution of $P_{i}$ as destruction increases. The important point is that extrapolation to complete destruction suggests that at that stage the $\mathrm{P}_{\mathrm{i}}$ concentration would be effectively zero. This indicates that $\mathrm{P}_{i}$ resonances are derived from the intracellular compartment and are not being received



Figure 7 Change in $\mathrm{pH}_{\mathrm{i}}$ from control, plotted against FVR

The curve represents $y=-0.0034-(0.1687 \cdot \ln \mathrm{FVR})$. Data are from the same studies as in Figure 2 (right panel). See the text for further details.

either from the extracellular space or from any residual intracellular $\mathrm{P}_{\mathrm{i}}$ in the region of destruction, thus validating the use of the chemical shift of $\mathrm{P}_{\mathrm{i}}$ for estimation of $\mathrm{pH}_{\mathrm{i}}$ in the unaffected zone. There is no evidence that the best fit to the data in Figure 5 is other than linear, implying [Appendix 1, eqn. (4), and Figure 3 , upper panel] that $\mathrm{P}_{i}$ does not vary appreciably along the lobular radius.

\section{ATP}

Figure 6 shows the mean ATP concentration in the unaffected zone of the lobules after various degrees of liver destruction. The fitted curve is sigmoidal, indicating a biphasic distribution of ATP along the lobular radius, with concentrations being highest in the mid-zone [see Appendix 1, eqn. (4), and Figure 3, upper panel].

\section{$\mathrm{pH}_{\mathrm{i}}$}

Figure 7 shows the change in mean $\mathrm{pH}_{\mathrm{i}}$ in the unaffected zone (compared with the control) as the degree of destruction was increased. The $\mathrm{pH}_{\mathrm{i}}$ before digitonin treatment was $7.346 \pm 0.021$ $(n=24)$. There was little change in $\mathrm{pH}_{\mathrm{i}}$ until $60 \%$ of the lobular volume had been destroyed. $\mathrm{The}_{\mathrm{pH}}$ then increased progressively steeply. The value of $\mathrm{pH}_{\mathrm{i}}$ (7.9) at very low FVR represents that in the most extreme periportal cells. However, the position of the peak of the $\mathrm{P}_{\mathrm{i}}$ resonance as the FVR changes cannot be simply regarded as an indicator of the true mean $\mathrm{pH}_{\mathrm{i}}$ of the remaining viable cells at each FVR, because that resonance is a composite of contributions from 'shells' (see Appendix 1) of differing volume and $\mathrm{pH}_{\mathrm{i}}$, with consequently differing chemical shifts. The procedure of Appendix 1 cannot, therefore, be applied to determine point-by-point $\mathrm{pH}_{\mathrm{i}}$ along the lobular radius. However, it can be inferred from Figure 7 that $\mathrm{pH}_{\mathrm{i}}$ falls from 7.9 periportally to $<7.4$ by $16 \%$ along the radius centripetally, and to $<7.35$ in the perivenous region. 


\section{DISCUSSION}

The salient features of the technique we describe are: (i) the observations are made in perfused liver rather than isolated hepatocytes, (ii) there is a precise measurement of the proportion of liver unaffected by digitonin, and (iii) it permits detailed mapping of metabolite concentration or function within the hepatic lobule.

The technique has a number of advantages over the conventional approach of preparing isolated cells from the postdigitonin liver remnant. First, it cannot be assumed that isolated cells, even from a specific zone, retain all properties, including metabolite concentrations, that they had in situ, since (i) their polarity is destroyed, (ii) a much greater cell surface area is presented to the extracellular environment, (iii) properties related to the flow rate of perfusate and to the order of cells along the sinusoid are lost, and (iv) other changes may have occurred during the isolation process [14]. Secondly, it is possible to determine substrate uptake and output of the periportal zone without modification by passage through a functioning centrilobular zone. Thirdly, it is possible to determine whether intracellular changes, e.g. in $\mathrm{pH}$ and adenine nucleotides, measured in whole liver give a misleading picture due to averaging over different zones of the lobule. Fourthly, it should be possible to determine whether a metabolic process is completed in one zone of the lobule, or whether part of the process is achieved proximally with the remainder being dependent on the passage of intermediate metabolites from the proximal zone to more distal sites. Finally, it would be virtually impossible, except by using very large numbers of livers, to produce the detailed maps illustrated here using the isolated cell technique, which has poor resolution. In the latter technique, the source of cells is determined by marker enzymes; although gradients of enzyme content exist along the length of the lobular radius, the precision of the marker enzyme technique is blurred in, say, a periportal preparation by the admixture of cells with high enzyme content near the portal tracts with cells of lower content further down the radius. The quantitative histomorphometry technique gives a precise measure of the remnant volume proportion in each liver studied; its precision is due to the very sharp demarcation between cells affected and unaffected by digitonin.

We have chosen always to use retrograde destruction, followed by antegrade perfusion. In this way, the remnant cells perfused are never in contact with any effluent derived from the region of damaged cells. Furthermore, if antegrade destruction had been followed by antegrade perfusion, the unaffected cells would have been supplied by perfusate which had not been modified by passage past more proximal cells, an unphysiological situation. It is, however, important to consider the possibility of very small quantities of digitonin, which may escape titration by the affected cells, reaching the histologically [3] and ultrastructurally [4] unaffected cells and affecting their metabolic function. Such a 'digitonin effect' has been found for glycogen synthesis, but not for gluconeogenesis, protein synthesis or Trypan Blue exclusion [8]; however, others have been unable to detect a digitonin effect for glycogen [15]. In our studies gluconeogenesis from lactate was stable over the time period examined, even at high levels of destruction, so progressive deterioration was not occurring. Nevertheless, the possibility of such effects cannot be entirely excluded.

Although the prime purpose of the present studies was to establish the method, some of the results require further comment. The predominance of gluconeogenesis from lactate in the periportal zone is expected $[1,3,5,15,16]$. Those previous studies, however, were not able to determine the detailed quantitative distribution of function demonstrated in Figure 2. Using the present technique, curves of the type shown in Figure 3 may be compared under different conditions of substrate and hormonal provision and in different nutritional states. Thus the curves of the distribution of gluconeogenesis for both 1.5 and $6 \mathrm{mM}$ lactate as substrate are not statistically different, and gluconeogenesis ceases at approximately the same relative distance along the lobular radius. The increased rate of gluconeogenesis seen at $6 \mathrm{mM}$ compared with $1.5 \mathrm{mM}$ lactate is therefore due entirely to a greater absolute rate of gluconeogenesis within the same periportal cells, and not to an extension of the process to cells more perivenously located. The cessation of gluconeogenesis by $50 \%$ down the radius is not due to substrate exhaustion by uptake into more proximally located cells.

The striking alkalinization of periportal cells compared with more distal ones in the lobule has not been described previously. There have been many studies of hepatic $\mathrm{pH}_{\mathrm{i}}$, using methods such as indicators (e.g. [10]) or ${ }^{31}$ P-NMR (e.g. [19]), on whole liver, either perfused or in situ, which produce a mean value, but these techniques could clearly obscure major intralobular variations such as seen in Figure 7. The narrowing of the line-width of the $\mathrm{P}_{\mathrm{i}}$ resonance is likely to be related to the decrease in $\mathrm{pH}_{\mathrm{i}}$ heterogeneity as the degree of destruction increases. We speculate that the very alkaline $\mathrm{pH}$ in the periportal region is due to the proton-consuming effect of gluconeogenesis from the lactate ion [10]. There is evidence $[10,19,20]$ that, at low and normal levels of blood lactate, a substantial fraction of lactate enters the hepatocyte as the ion rather than as the undissociated acid. It should be noted that the conditions of the present studies are non-physiological in the sense that lactate is the sole substrate. Further detailed studies with physiological substrate mixtures are required, and could demonstrate different patterns of metabolite concentrations. Some caution should be exercised with regard to the highest levels of $\mathrm{pH}_{\mathrm{i}}$ recorded, since these are near the upper end of the phosphate titration curve. There is clearly a variation of ATP concentration along the sinusoid; this may have implications for hepatic energetics and metabolic regulation, previously studied using mean values from direct analysis or ${ }^{31} \mathrm{P}$ NMR of whole liver [21]. The lower periportal ATP concentration compared with that in the mid-zone of the lobule could be attributable to ATP consumption by gluconeogenesis. Further studies, including the use of inhibitors of gluconeogenesis, are required to test this hypothesis and to establish the origin of the periportal alkalinity discussed above. Finally, the method is likely to be generally applicable to a wide range of hepatic functions, and to concentrations of metabolites other than those examined here.

A superficially surprising feature is the ease with which liver perfusion can be maintained after extensive cellular destruction by digitonin. However, an analogous situation occurs in clinical viral hepatitis, in which there is centrilobular zonal necrosis of varying extent. The reticulin framework of the liver is, nevertheless, preserved and allows continued blood flow through the affected liver, and subsequent regeneration preserving the original architecture. Presumably, similar preservation of the framework after digitonin treatment is responsible for the ability to maintain perfusion. These considerations suggest that NMR could be used clinically to study the progress of regeneration in acute hepatitis and factors that affect it. Such studies would require noninvasive estimates of the degree of hepatic necrosis.

This work was supported by the U.K. Medical Research Council. We are grateful to Professor D. M. Williams and Dr. Alan Cruchley for histological and histomorphometric advice and equipment, and to the University of London Intercollegiate Research Service at Queen Mary and Westfield College for NMR facilities. 


\section{REFERENCES}

1 Jungermann, K. and Katz, N. (1989) Physiol. Rev. 69, 708-764

2 Quistorff, B. and Grunnet, B. (1985) Biochem. J. 226, 289-297

3 Quistorff, B. and Romert, P. (1989) Histochemistry 92, 487-498

4 Racine, L., Scoazec, J., Moreau, A., Bernuau, D. and Feldmann, G. (1993) J. Histochem. Cytochem. 7, 991-1001

5 Quistorff, B. (1985) Biochem. J. 229, 221-226

6 Pösö, A. R., Penttilä, K. E., Suolinna, E. and Lindros, K. O. (1986) Biochem J. 239, 263-267

7 Tosh, D., Alberti, K. G. M. M. and Agius, L. (1988) Biochem. J. 256, 197-204

8 Chen, K. S. and Katz, J. (1988) Biochem. J. 255, 99-104

9 Guder, W. G. and Schmidt, U. (1976) Hoppe-Seyler's Z. Physiol. Chem. 357, 1793-1800

10 Cohen, R. D., Iles, R. A., Barnett, D., Howell, M. E. O. and Strunin, J. (1971) Clin. Sci. 41, 159-170
11 Krebs, H. A. and Henseleit, K. (1932) Hoppe-Seyler's Z. Physiol. Chem. 210, 33-36

12 Hohorst, H. J. (1962) in Methods of Enzymatic Analysis (Bergmeyer, H. U., ed.), pp. 266-270, Academic Press, New York

13 Matthews, J. N. S. and Altman, D. G. (1989) Br. Med. J. 300, 230-235

14 Guzman, M., Bijleveld, C. and Geelen, M. J. H. (1995) Biochem. J. 311, 853-860

15 Agius, L., Peak, M. and Alberti, K. G. M. M. (1990) Biochem. J. 266, 91-102

16 Agius, L. and Tosh, D. (1990) Biochem. J. 271, 387-391

17 Reference deleted

18 Reference deleted

19 Beech, J. S., Williams, S. R., Cohen, R. D. and Iles, R. A. (1989) Biochem. J. 263, $737-744$

20 Fafournoux, P., Démigné, C. and Rémésy, C. (1985) J. Biol. Chem. 260, 292-299

21 Iles, R. A., Stevens, A. N., Griffiths, J. R. and Morris, P. G. (1985) Biochem. J. 229, $141-151$

\section{APPENDIX 1}

\section{Derivation of plots of function or metabolite concentration at points along the lobular radius from the centre}

For function, it is assumed that on average the lobules are spherical, with mean radius $R$, and with a radial distribution of cords of hepatocytes of uniform size. $r$ is the mean radius of the destroyed volume, $x$ is the fractional volume (FVR) of the unaffected zone surrounding the destroyed volume and $F$ is the overall function remaining, relative to the pre-digitonin value. A suitable function $F=f(x)$ is fitted to the data as in Figure 2; $y_{x}$ is the function of a volume element of cells of radial width $r$ at the destruction boundary at FVR $=x$. Suppose the degree of destruction had been marginally less, with the result that the function of this volume element now has to be included. All cells in the same concentric shell (e.g. one cell thick) as the index unit volume now contribute to the overall function. The function $F$ of that shell is $4 \pi r^{2} \cdot \delta r \cdot y_{x}$. Therefore:

$\mathrm{d} F / \mathrm{d} r=4 \pi r^{2} y_{x}$

Since $x=1-(r / R)^{3}$ and $\mathrm{d} F / \mathrm{d} r=(\mathrm{d} F / \mathrm{d} x)(\mathrm{d} x / \mathrm{d} r)$, then:

$-3 R^{-3}(\mathrm{dF} / \mathrm{dx})=4 \pi y_{x}$
Now, suppose that $y_{0}$ is the function of a unit volume at the extreme periportal end $(x=0)$. Then, by substituting $y_{0}$ and $x=0$, or $y_{x}$ and $x=x$, in eqn. (2) and dividing, we get:

$y_{x} / y_{0}=(\mathrm{d} F / \mathrm{d} x)_{x=x} /(\mathrm{d} F / \mathrm{d} x)_{x=0}$

The differentials are obtained from the fitted curve and plotted against $x$ or $r$.

When considering the concentration of a metabolite, e.g. [ATP] or $\left[\mathrm{P}_{\mathrm{i}}\right]$, a curve is fitted to $Q=f_{1}(x) . Q$ is the total amount of metabolite, relative to that pre-digitonin, in the undestroyed volume, determined by NMR peak area. Proceeding as above for function, we get:

$z_{x} / z_{m}=(\mathrm{d} Q / \mathrm{d} x) /(\mathrm{d} Q / \mathrm{d} x)_{x=x_{m}}$

where $z_{x}$ is the concentration of a particular metabolite in a nominal intact unit volume at the destruction boundary, and $z_{m}$ is the mean metabolite concentration at the smallest FVR, $x_{m}(\leqslant 0.1)$, at which it is possible to get a satisfactory NMR measurement.

where $\boldsymbol{h}=\left(\begin{array}{l}\boldsymbol{h}_{1} \\ \boldsymbol{h}_{2}\end{array}\right)$ as above, $\boldsymbol{h}^{\prime}$ is its transpose and the matrix $\boldsymbol{V}$ is the variance-covariance matrix of the estimated parameters $b$ and $c$.

The parameter estimates from the data are:

$b=0.12793 ; \quad c=0.03469$;

$\boldsymbol{V}=\left(\begin{array}{ll}\boldsymbol{V}_{11} & \boldsymbol{V}_{12} \\ \boldsymbol{V}_{12} & \boldsymbol{V}_{22}\end{array}\right)=\left(\begin{array}{cc}0.000837 & 0.000227 \\ 0.000227 & 0.0000617\end{array}\right)$

The $95 \%$ confidence intervals for $y^{\prime}(x) / y^{\prime}\left(x_{m}\right)$ are given by:

$\left[y^{\prime}(x) \pm 1.96 s(x, b, c)\right] / y^{\prime}\left(x_{m}\right)$

\section{REFERENCES}

1 Hinkley, D. V. and Reid, N. (1991) in Statistical Theory and Modelling (Hinkley, D. V., Reid, N. and Snell, E. J., eds.), p. 27, Chapman and Hall, London

$s(x, b, c)=\left(\boldsymbol{h}^{\prime} \boldsymbol{V h}\right)^{\frac{1}{2}}=\left[h_{1}\left(h_{1} v_{11}+h_{2} v_{12}\right)+h_{2}\left(h_{1} v_{12}+h_{2} v_{22}\right)\right]^{\frac{1}{2}}$ 